\title{
Using Photoactive N-Heterocyclic Carbenes Monolayers to Identify the Influence of Surface Proximity on Photoswitching Activity
}

Shahar Dery ${ }^{+}$, Israel Alshanski ${ }^{+}$, Evgeniy Mervinetsky ${ }^{+}$, Daniel Feferman, Shlomo Yitzchaik*, Mattan Hurevich*, and Elad Gross * Institute of Chemistry and The Center for Nanoscience and Nanotechnology, The Hebrew University, Jerusalem 91904, Israel

\footnotetext{
${ }^{+}$These authors equally contributed to this work

*Email address: shlomo.yitzchaik@mail.huji.ac.il; mattan.hurevich@mail.huji.ac.il; elad.gross@mail.huji.ac.il
} 


\section{Abstract}

Self-assembly of photoresponsive molecules is a robust technology for reversibly tuning the chemical and electronic properties of functional materials. In most systems the photoactive group is separated from the surface by a spacer and thus the photoresponsiveness does not benefit from interactions with the metal. Herein, the impact of metal photoactive-group interactions on photoswitchability and surface potential were probed by self-assembly of N-heterocyclic carbene molecules (NHCs) that were functionalized with stilbene group directly on their imidazole ring. Stilbene-NHCs that were adsorbed on weakly interacting $\mathrm{Au}$ surface accumulated a vertical orientation, as identified by FTIR measurements. This positioning enabled structural flexibility and high photoisomerization efficiency that induced reversible changes in surface potential. Stilbene-NHCs that were anchored on Pt film accumulated flatlying adsorption geometry due to strong metal-adsorbate interactions. These interactions limited the structural flexibility of the stilbene groups and induced deteriorated photoswitchability that led to lower photoinduced changes in surface potential. While stronger metal-adsorbate interactions hindered the photo-induced isomerization yield of stilbene, these interactions prompted the cis-to-trans thermalinduced isomerization rate, which was an order of magnitude higher on Pt than on Au.

Keywords: N-Heterocyclic Carbene; Self-assembled monolayers; Stilbene; Photoswitch 


\section{Introduction}

The use of light as a non-invasive stimulus and the wide chemical versatility of photoresponsive organic-based monolayers make these systems highly beneficial for photo-adjusting the surface properties of functional materials. ${ }^{1,2}$ These advantages led to the utilization of photoresponsive organic-based monolayers in the fields of nanoscience, ${ }^{3}$ electronics, ${ }^{4,5}$ sensing $^{6,7}$ and catalysis. ${ }^{8,9}$

Photoresponsive monolayers are constructed of two segments: i. photoactive group, such as azobenzenes ${ }^{10,11}$, stilbenes ${ }^{12}$ and spiropyrans ${ }^{13-16}$; ii. surface anchoring handle, which is frequently thiol-based. ${ }^{3}$ It should be noted that the metal or oxide surface on which the monolayer is anchored can potentially function as a third segment in the photoresponsive system and influence the photoswitchability. However, in most systems a lateral spacer separates the photoactive group from the surface anchoring handle and partially decouple the surface properties from that of the photoactive group (Scheme 1). ${ }^{3}$

\section{Previous works}

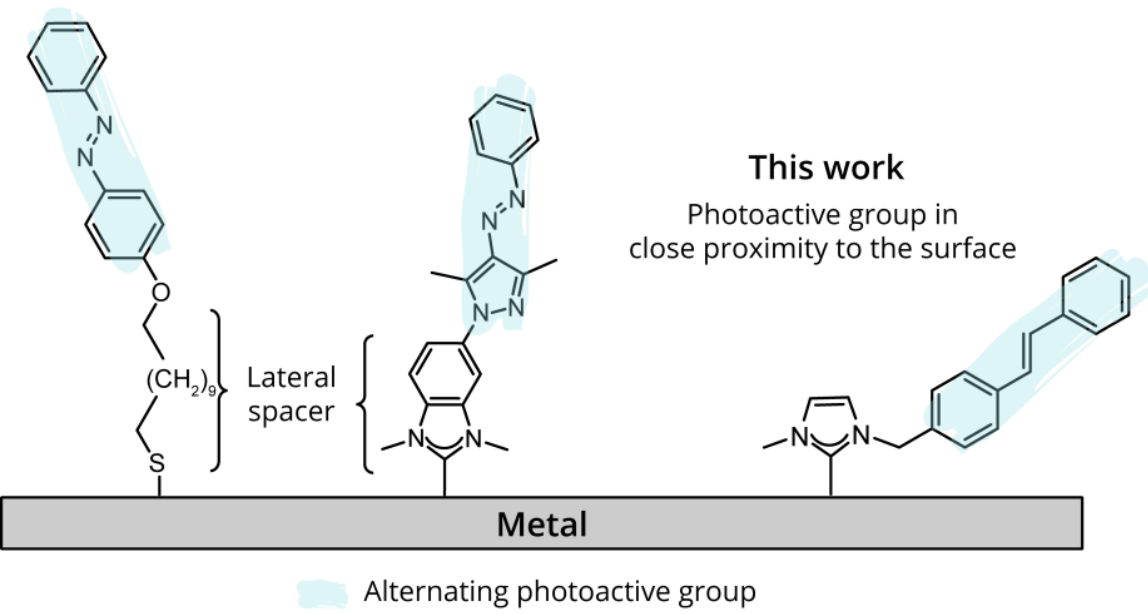

Scheme 1. Previous works displaying photoresponsive thiol- and NHC-based monolayers in which the photoactive group is separated from the surface using a lateral alkyl or benzimidazole spacer (left). In this work the photoactive group was positioned in proximity to the metal surface, yielding a metal-dependent coupling of the photoelectronic effect (right). 
It was recently demonstrated that $\mathrm{N}$-heterocyclic carbene molecules (NHCs) can be utilized as a surface anchoring group for the formation of photoresponsive monolayers by installing arylazopyrazole group on the benzimidazole backbone (Scheme 1). ${ }^{17}$ The metal-affinity of NHCs ${ }^{18-20}$ enabled to form robust photoresponsive NHCs-based monolayers with exceptional stability. ${ }^{17}$

Since NHCs are anchored to the surface through the carbene of the imidazole ring, $N$-substitution of the imidazole allows the positioning of a responsive group in high proximity to the metal. ${ }^{19,21-26}$ The ability to position chemically-reactive groups in proximity to the metal support enabled to utilize metal-adsorbate interactions for tuning the chemical reactivity of the addressable $\operatorname{group}^{21-23,25,26}$ and the metal properties, i.e. surface potential. ${ }^{24,27}$ In a similar way, $N$-substitution of the imidazole ring by photoactive groups can provide a way to position these groups in close proximity to the surface (Scheme 1) and tune the surface effect on photoreactivity while benefiting from the high thermal stability of surface-anchored NHCs. To the best of our knowledge, such system has not been reported yet.

Herein, a photoactive stilbene group was positioned directly on the imidazole scaffold, thus yielding NHC-based monolayer in which the photoresponsive group is located in close proximity to the metal surface (Scheme 1). Stilbene was chosen as the photoresponsive group since it enables evaluating the influence of surface proximity on both photo-induced trans-to-cis isomerization and thermal-induced cis-to-trans isomerisation. ${ }^{12,28,29}$ Reversible changes in the surface potential were detected following photoisomerization of the stilbene groups in stilbene-NHC monolayer that was anchored on $\mathrm{Au}$. Strong interaction between stilbene-NHC and Pt surface hindered the photoisomerization and reduced the photo-induced changes in surface potential. However, the strong interaction of stilbene-NHC with Pt led to an order of 
magnitude increase in thermal-induced cis-to-trans isomerization rate, in comparison to the rate measured on $\mathrm{Au}$, demonstrating the impact of strong metal-adsorbate interactions on isomerization kinetics.

\section{Results and discussion}

Photoactive NHC molecules were designed to include imidazole scaffold and photoswitchable stilbene functionality separated via a methylene group (Scheme 1). This design ensures high proximity of the stilbene to the metal surface while decoupling the two aromatic systems (imidazole and stilbene) by an $\mathrm{sp}^{3}$ carbon, which breaks their conjugation. The non-conjugated system provides an opportunity to exclusively measure the influence of photoinduced isomerization on surface properties. Stilbene-NHC adduct was synthesized via a one-step $S_{N} 2$ reaction, by exploiting the reactivity of the free nitrogen atom in the imidazole ring. ${ }^{30}$ The desired adduct, 1-p-methyl-trans-stilbene-3-methyl-1H-imidazolium chloride (stilbeneimidazolium, 1), was prepared at $84 \%$ yield by $\mathrm{N}$-substitution of $\mathrm{N}$-methylimidazole with 4-chloromethyl-trans-stilbene (Scheme 2, full synthetic details can be found in the SI, Fig. S1-S6). Stilbene-NHC was prepared by deprotonation of stilbeneimidazolium using $\mathrm{KO}^{t} \mathrm{Bu}$ in THF under inert conditions. The resulting stilbene-NHC was self-assembled on $\mathrm{Au}$ and Pt films that were evaporated on Si wafers.

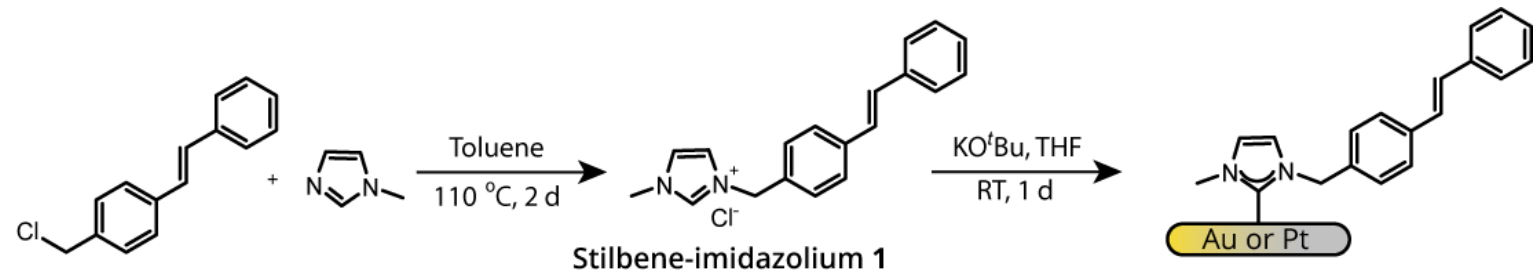

Scheme 2. Synthetic scheme for the preparation of 1-p-methylstilbene-3-methyl-1Himidazolium chloride (Stilbene-imidazolium, 1) from 4-chloromethylstilbene and $\mathrm{N}$-methylimidazole in toluene. Deprotonation led to the formation of stilbene-NHC that was self-assembled on Au and Pt surfaces. 
The presence of a surface-anchored monolayer on $\mathrm{Au}$ and $\mathrm{Pt}$ films was identified by N1s XPS measurements. While the N1s XPS spectrum of stilbene-imidazolium salt displayed a dominant peak centered at $402.0 \mathrm{eV}$ (Fig. 2a) the surface-anchored stilbene-NHC on Au and Pt exhibited a peak positioned at $400.0 \mathrm{eV}$ (Fig. 2b-c). ${ }^{21-23}$ The shift in the peak position following deprotonation was correlated to neutralization of the positive charge in stilbene-imidazolium and was identified as well in other NHC-based monolayers. ${ }^{17}$
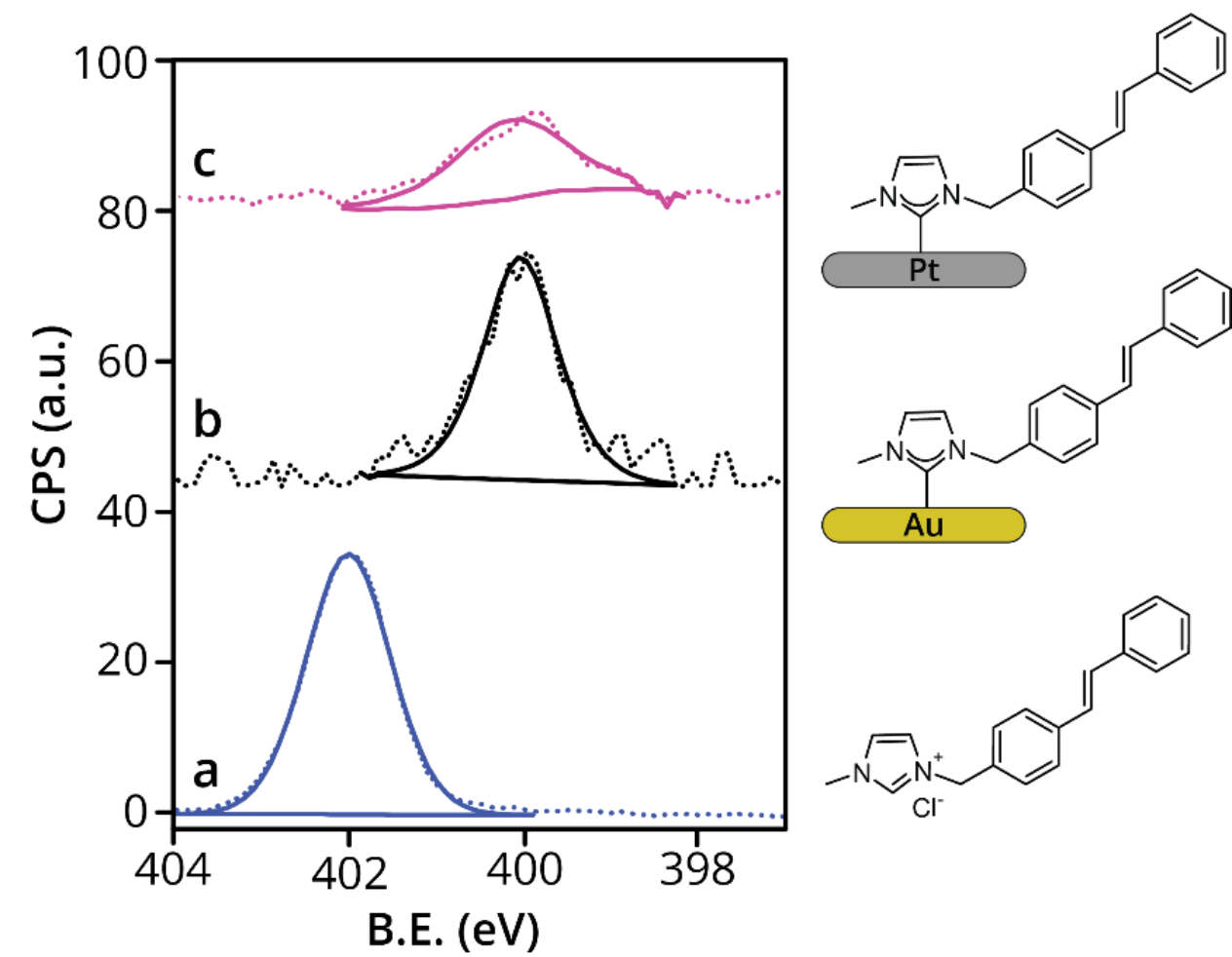

Figure 1. N1s XPS spectra of stilbene-imidazolium salt (a) and stilbene-NHC that was anchored on $\mathrm{Au}(\mathbf{b})$ and $\mathrm{Pt}(\mathbf{c})$ films.

Contact potential difference (CPD) measurements were performed to analyze the influence of stilbene-NHC coating on the work function (WF) values of $\mathrm{Au}$ and Pt. Surface-anchoring of stilbene-NHC on Au and Pt films changed their WF values by $0.36 \mathrm{eV}$ and $-0.25 \mathrm{eV}$, respectively. The decrease in the $\mathrm{WF}$ values results from the 
interface dipole that is formed between the NHC and the Au or Pt surfaces. ${ }^{27}$ The higher change in the surface potential of $\mathrm{Au}$, in comparison to $\mathrm{Pt}$, can be associated with the higher order of stilbene-NHC on $\mathrm{Au}^{21}$

The photoswitchability of surface-anchored stilbene-NHC and its impact on surface properties was examined by WF measurements under irradiation. Each cycle consisted of one-hour irradiation $(365 \mathrm{~nm})$ followed by relaxation without illumination. CPD measurements showed a photo-induced increase of $90 \pm 10 \mathrm{meV}$ in WF values after irradiation of stilbene-NHC/Au (Fig. 2a). The increase in the WF values following illumination was correlated to photo-induced trans-to-cis isomerization of the stilbene group, which lowered the molecular dipole pointing towards the surface. ${ }^{1,11,13,31}$ Thermal-induced relaxation (without illumination) yielded a decrease in the WF values, correlated to cis-to-trans isomerization of the stilbene group. ${ }^{29}$ The isomerization of stilbene-NHC on Au led to WF changes that were in the same range of those measured for arylazopyrazole-based NHC. ${ }^{17}$ The reversibility of the cis-to-trans isomerization was shown over several cycles, with no notable decrease in efficiency.

a

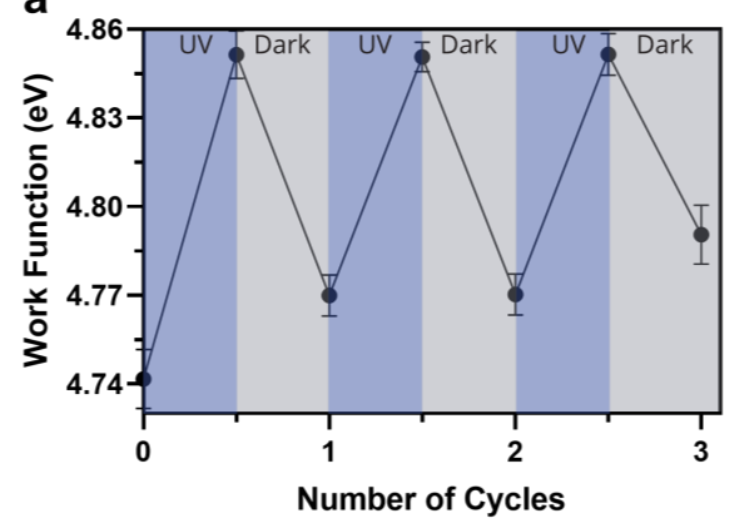

b

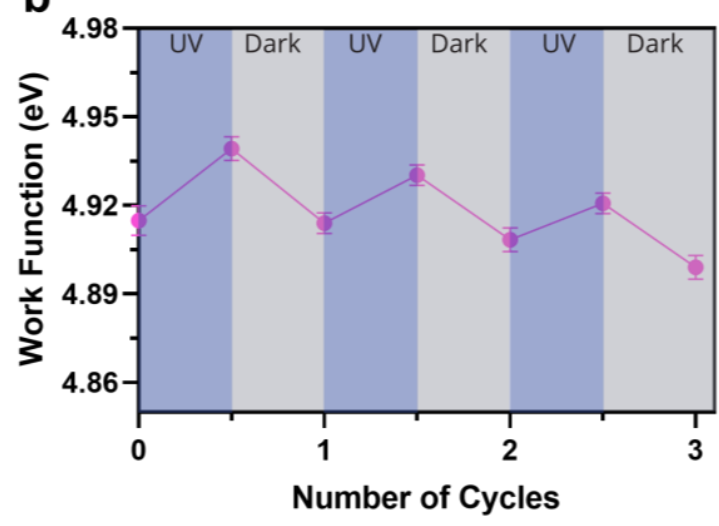

Figure 2. WF measurements under UV irradiation (365 nm) of $\mathrm{Au}(\mathbf{a})$ and $\mathrm{Pt}(\mathbf{b})$ films that were functionalized with stilbene-NHC (connecting lines were added as a guide to the eye). Error bars represent the SD values of three samples. 
Photo-induced WF changes of $37 \pm 10 \mathrm{meV}$ were measured for stilbene-NHC that was anchored on Pt film (Fig. 2b). These values are 2.5 times lower than the values measured for stilbene-NHC/Au. Thus, although the surface density of stilbene-NHC on Au was comparable to the density on $\mathrm{Pt}$, the photo-induced impact on WF was quenched once stilbene-NHC was anchored on Pt. The deteriorated photo-induced $\Delta \mathrm{WF}$ values measured for stilbene-NHC/Pt can be correlated with the strong interaction of stilbene-NHC with the Pt film. These interactions can also explain the gradual decrease in the WF values that was measured over several cycles.

Photo-inert dimethyl functionalized-NHCs were deposited on Au and Pt films and their photo-induced influence on the WF were measured. Light-to-dark WF changes of $6 \pm 2$ and $2 \pm 1 \mathrm{meV}$ were measured for $\mathrm{Au}$ and Pt films that were modified with dimethyl functionalized-NHCs, respectively (Fig. S7). These results indicate that the photo-induced changes in the WF values of stilbene-NHC were correlated with the presence of a photoswitchable stilbene group and were not induced by the imidazole scaffold.

In-situ polarization modulation infrared reflection absorption spectroscopy (PMIRRAS) measurements of stilbene-NHC/Au and stilbene-NHC/Pt (Fig. 3a and 3b, respectively) were conducted under illumination and after thermal relaxation (purple and black coloured spectra, respectively). The high sensitivity of PM-IRRAS measurements to dipoles that are perpendicular to the surface enables to evaluate the influence of illumination on the positioning of surface-anchored stilbene-NHC on $\mathrm{Au}$ and Pt surfaces. 

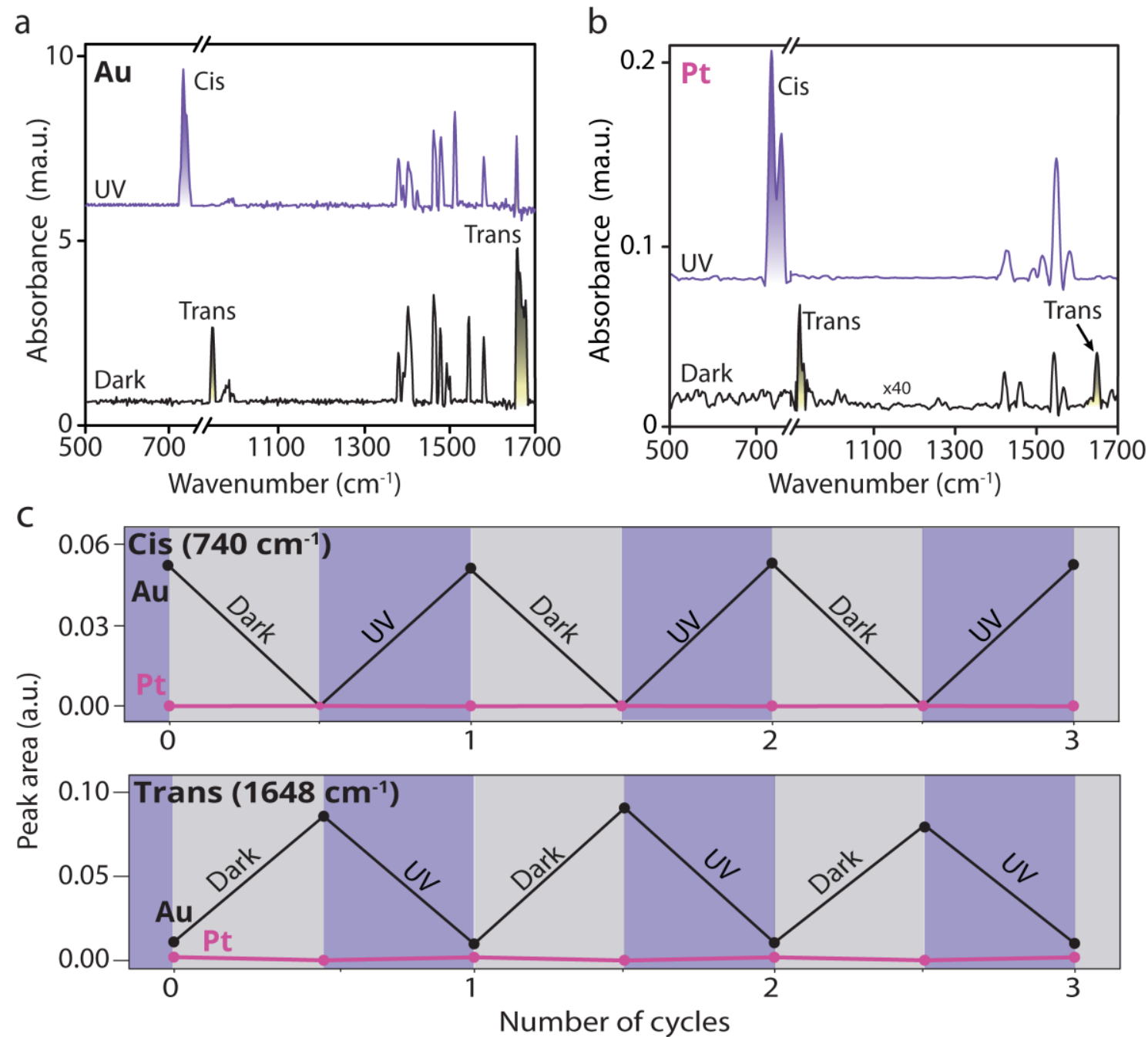

Figure 3. In-situ PM-IRRAS measurements of stilbene-NHC that was anchored on $\mathrm{Au}$ (a) and Pt (b) films. Black and purple spectra were acquired under UV-irradiation and dark, respectively. c. Peak area analysis of cis- and trans-correlated vibrations (734 and $1648 \mathrm{~cm}^{-1}$, respectively) of stilbene-NHC that was anchored on Au and Pt films (black and pink-colored data points, respectively) under light-dark cycles.

Illumination led to the appearance of a peak at $740 \mathrm{~cm}^{-1}$ on both $\mathrm{Au}$ and Pt surfaces. This peak was attributed to the $c i s$-stilbene isomer ${ }^{29,32,33}$ and was not detected under dark conditions. Additionally, surface illumination eliminated two vibrational signatures at 947 and $1648 \mathrm{~cm}^{-1}$, which were assigned to $\mathrm{C}-\mathrm{H}$ bending and $\mathrm{C}=\mathrm{C}-\mathrm{H}$ stretch of the trans double bond moiety, respectively. The vibrational signals in the 
1300-1600 $\mathrm{cm}^{-1}$ range, which were assigned to the imidazole ring, were not significantly modified following illumination. This result indicates that the imidazole ring did not change its positioning during the light-dark cycles. Thus, the vibrational spectroscopy measurements confirmed that the illumination-induced changes in the WF values were due to stilbene isomerization and were not connected to changes in the imidazole ring position.

The amplitudes of the cis- and trans-correlated vibrational peaks were 20 fold higher on $\mathrm{Au}$ than on Pt. Since the surface density of stilbene-NHC on $\mathrm{Au}$ and $\mathrm{Pt}$ is comparable, as identified by XPS measurements (Fig. 1), the vast differences in the vibrational amplitude were correlated to variations in the orientation of stilbene-NHC. The weak vibrational signals of stilbene-NHC on Pt indicate that the surface-anchored molecules were oriented in a close to flat-lying position. ${ }^{21,23}$ The strong interaction of stilbene-NHCs with the underlying Pt surface led to preference of a flat-laying position that limited the structural flexibility of the stilbene group. The limited structural flexibility of stilbene-NHC on $\mathrm{Pt}$ circumvented the photo-induced isomerization and induced lower changes in the work-function values, in comparison to those measured on $\mathrm{Au}$.

The reversible appearance and disappearance of a cis-related peak $\left(740 \mathrm{~cm}^{-1}\right)$ under illumination and dark conditions, respectively, and the appearance and disappearance of a trans-related peak (1648 $\left.\mathrm{cm}^{-1}\right)$ under dark and illumination conditions, respectively, were monitored in three consecutive dark-light cycles (Fig. 3c). High reversibility of photo-isomerization was detected for both stilbene-NHC/Au and stilbene-NHC/Pt (Fig. 3c, black- and pink-coloured data points, respectively). However, the amplitudes of both cis- and trans-related vibrational signals on Pt were 20 fold lower than those detected on $\mathrm{Au}$, demonstrating the restricted 
photoisomerization of stilbene-NHC that was anchored on Pt and correlated to the flat lying position of the molecules.

The influence of surface proximity on the thermal-induced cis-to-trans isomerization rate was studied by comparing the WF changes in stilbene-NHC/Au and stilbeneNHC/Pt (Fig. 4). A much faster decay in the WF values was detected for NHCstilbene/Pt than for NHC-stilbene/Au. The WF values were fit to exponential decay and half-life time values of 889 and $89 \mathrm{sec}$ were extracted for NHC-stilbene/Au and NHC-stilbene/Pt, respectively. The 10-fold higher decay rate for NHC-stilbene on Pt, in comparison to the values measured on $\mathrm{Au}$, indicate that cis-to-trans isomerization rate was facilitated on Pt. It is hypothesized that the higher isomerization rate is the result of stronger coupling between NHC-stilbene and Pt support that enhanced the thermal-induced isomerization efficiency.

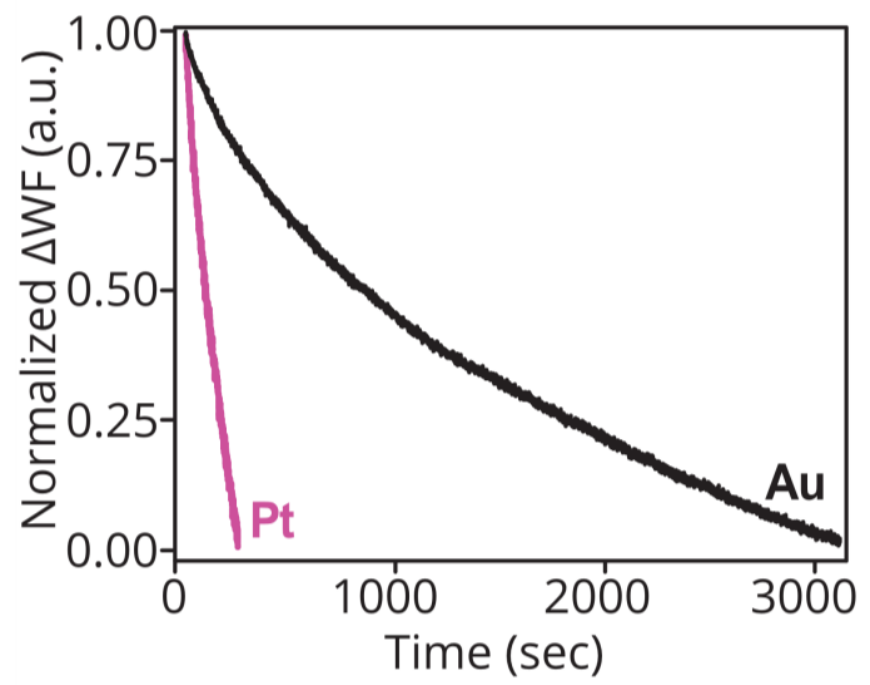

Figure 4. $\triangle \mathrm{WF}$ decay curves upon thermal relaxation of stilbene-NHC/Au (black) and stilbene-NHC/Pt (pink). 


\section{Conclusion}

$\mathrm{N}$-substituted NHC bearing a stilbene group was synthesized and anchored on Au and Pt films, enabling to position the photoactive group in close proximity to the metal surface. In-situ PM-IRRAS and WF measurements provided holistic view of the influence of metal-adsorbate interactions on the yield and rate of stilbene isomerization and its impact on surface properties. Stilbene-NHCs that were anchored on the more inert and weakly interacting Au surface accumulated a vertical orientation, which allowed structural flexibility and high isomerization efficiency. The molecular orientational order of stilbene-NHC on $\mathrm{Au}$ and the high photoisomerization yield resulted in reversible photoinduced changes of $90 \pm 10 \mathrm{meV}$ in WF value. Strong metal-adsorbate interactions between stilbene-NHCs and the supporting Pt surface led to flat-lying adsorption geometry that limited the structural flexibility and photoswitchability of stilbene. Strong metal-adsorbate interactions, on the other hand, facilitated the thermal-induced cis-to-trans isomerization rate, characterized with an order of magnitude higher rate on Pt than on Au. The presented results identify the key impact of metal-adsorbate interactions on photoisomerization, demonstrating that strong metal-adsorbate interactions limit the isomerization yield but can increase the isomerization rate. Photoresponsive monolayers can be therefore designed with enhanced yield or rate by utilizing metal-adsorbate interactions.

\section{Acknowledgments}

This research was supported by the European Research Council (ERC) under the European Union's Horizon 2020 research and innovation program (Grant Agreement

No. 802769, ERC Starting Grant "MapCat”). S.Y. thanks the Binjamin H. Birstein 
Chair in Chemistry. S.D. acknowledges the Israeli Ministry of Energy and the Azrieli

Foundation for the award of an Azrieli Fellowship. I.A. is supported by Hebrew

University Center for Nanoscience and Nanotechnology PhD. Scholarship.

\section{References}

(1) Orgiu, E.; Samori, P. Adv Mater 2014, 26, 1827.

(2) Russew, M. M.; Hecht, S. Adv Mater 2010, 22, 3348.

(3) Bian, T.; Chu, Z. L.; Klajn, R. Adv Mater 2020, 32.

(4) Wang, Y.; Cheng, H. P. Phys Rev B 2012, 86.

(5) Zhang, C.; Du, M. H.; Cheng, H. P.; Zhang, X. G.; Roitberg, A. E.; Krause, J. L. Phys Rev Lett 2004, 92.

(6) He, Y.; Wang, R.; Sun, C. G.; Liu, S. F.; Zhou, J. X.; Zhang, L. X.; Jiao, T. F.; Peng, Q. M. Acs Omega 2020, 5, 3689.

(7) Kim, K. T.; Il Moon, N.; Kim, H. K. Sensor Actuat a-Phys 2010, 160, 19.

(8) Dorel, R.; Feringa, B. Chem Commun 2019, 55, 6477.

(9) Wei, Y. H.; Han, S. B.; Kim, J.; Soh, S. L.; Grzybowski, B. A. J Am Chem Soc 2010, $132,11018$.

(10) Bleger, D.; Ciesielski, A.; Samori, P.; Hecht, S. Chem-Eur J 2010, 16, 14256.

(11) Delorme, N.; Bardeau, J. F.; Bulou, A.; Poncin-Epaillard, F. Langmuir 2005, 21, 12278.

(12) Zhang, J.; Whitesell, J. K.; Fox, M. A. Chem Mater 2001, 13, 2323.

(13) Kim, M.; Safron, N. S.; Huang, C. H.; Arnold, M. S.; Gopalan, P. Nano Lett 2012, 12, 182.

(14) Suda, M.; Kameyama, N.; Suzuki, M.; Kawamura, N.; Einaga, Y. Angew Chem Int Edit 2008, 47, 160.

(15) Ikbal, M.; Balogh, D.; Mervinetsky, E.; Sfez, R.; Yitzchaik, S. J Phys Chem C 2017, $121,27176$.

(16) Kundu, P. K.; Das, S.; Ahrens, J.; Klajn, R. Nanoscale 2016, 8, 19280.

(17) Nguyen, D. T.; Freitag, M.; Gutheil, C.; Sotthewes, K.; Tyler, B. J.; Bockmann, M.; Das, M.; Schluter, F.; Doltsinis, N. L.; Arlinghaus, H. F.; Ravoo, B. J.; Glorius, F. Angew Chem Int Edit 2020, 59, 13651.

(18) Crudden, C. M.; Horton, J. H.; Ebralidze, I. I.; Zenkina, O. V.; McLean, A. B.; Drevniok, B.; She, Z.; Kraatz, H. B.; Mosey, N. J.; Seki, T.; Keske, E. C.; Leake, J. D.; Rousina-Webb, A.; Wu, G. Nat Chem 2014, 6, 409.

(19) Ranganath, K. V. S.; Kloesges, J.; Schafer, A. H.; Glorius, F. Angew Chem Int Edit 2010, 49, 7786.

(20) Zhukhovitskiy, A. V.; Mavros, M. G.; Van Voorhis, T.; Johnson, J. A. J Am Chem Soc 2013, 135, 7418 .

(21) Dery, S.; Berg, I.; Kim, S.; Cossaro, A.; Verdini, A.; Floreano, L.; Toste, F. D.; Gross, E. Langmuir 2020, 36, 697.

(22) Dery, S.; Kim, S.; Haddad, D.; Cossaro, A.; Verdini, A.; Floreano, L.; Toste, F. D.; Gross, E. Chem Sci 2018, 9, 6523.

(23) Dery, S.; Kim, S.; Tomaschun, G.; Berg, I.; Feferman, D.; Cossaro, A.; Verdini, A.; Floreano, L.; Kluner, T.; Toste, F. D.; Gross, E. J Phys Chem Lett 2019, 10, 5099.

(24) Dery, S.; Kim, S.; Tomaschun, G.; Haddad, D.; Cossaro, A.; Verdini, A.; Floreano, L.; Kluner, T.; Toste, F. D.; Gross, E. Chem-Eur J 2019, 25, 15067.

(25) Levratovsky, Y.; Gross, E. Faraday Discuss 2016, 188, 345. 
(26) MacLeod, M. J.; Goodman, A. J.; Ye, H. Z.; Nguyen, H. V. T.; Van Voorhis, T.; Johnson, J. A. Nat Chem 2019, 11, 57.

(27) Kim, H. K.; Hyla, A. S.; Winget, P.; Li, H.; Wyss, C. M.; Jordan, A. J.; Larrain, F. A.; Sadighi, J. P.; Fuentes-Hemandez, C.; Kippelen, B.; Bredas, J. L.; Barlow, S.; Marder, S. R. Chem Mater 2017, 29, 3403.

(28) Repinec, S. T.; Sension, R. J.; Szarka, A. Z.; Hochstrasser, R. M. J Phys Chem-Us 1991, 95, 10380.

(29) Waldeck, D. H. Chem Rev 1991, 91, 415.

(30) Kaiser, M.; Leitner, S. P.; Hirtenlehner, C.; List, M.; Gerisch, A.; Monkowius, U. Dalton T 2013, 42, 14749.

(31) Lu, J. Z.; Lipatov, A.; Vorobeva, N. S.; Muratov, D. R. S.; Sinitskii, A. Adv Electron Mater 2018, 4.

(32) Meic, Z.; Gusten, H. Spectrochim Acta A 1978, 34, 101.

(33) Pecile, C.; Lunelli, B. Can J Chemistry 1969, 47, 243. 